\title{
Erratum to: In rDNS We Trust: Revisiting a Common Data-Source's Reliability
}

Tobias Fiebig, Kevin Borgolte, Shuang Hao, Christopher Kruegel,

Giovanni Vigna, and Anja Feldmann

\author{
Erratum to: \\ Chapter "In rDNS We Trust: Revisiting a Common \\ Data-Source's Reliability" in: R. Beverly et al. (Eds.): \\ Passive and Active Measurement, LNCS 10771, \\ https://doi.org/10.1007/978-3-319-76481-8_10
}

In the original version of this chapter, coloring was off in the in-addr.arpa part of Fig. 6a on page 141. IPv4 rDNS zones returning SERVFAIL were illustrated using the beige reserved for dynamic zones in the IPv6 graphs, instead of the correct wine-red used for SERVFAIL zones. In Section 5.3 on page 139, we reported that we used an ip6.arpa rDNS dataset collected between March 26th, 2017 01:04 UTC to March 30th, 2017 10:49 UTC. However, we collected the dataset we analyzed between April 22nd, 2017 04:07 UTC and April 25th, 2017 10:15 UTC. No incorrect data was reported, however, and the results remain valid. The original chapter has been corrected.

We thank our fellow researchers Robert Beverly from the Naval Postgraduate School and Oliver Gasser from TU Munich for pointing out these mistakes. They would not have been discovered without the publication of our collected dataset. Therefore, we would like to take this opportunity to plead in favor of Open Data in the field of network measurements.

\footnotetext{
The updated online version of this chapter can be found at https://doi.org/10.1007/978-3-319-76481-8_10 